\title{
Influencia de la adopción de tecnologías de información en el desempeño organizacional*
}

\author{
Leslier Valenzuela Fernández \\ Facultad de Economía y Negocios \\ Universidad de Chile \\ lvalenzuela@unegocios.cl
}

Carolina Martínez Troncoso

Facultad de Economía y Negocios

Universidad Finis Terrae

cmartinezt@uft.cl

\begin{abstract}
This research aims to (1) investigate the factors that influence the adoption of IT (2) study the possible influences between IT adoption, organizational performance and perception of quality service by the employees (3) provide empirical evidence through the testing of a descriptive model based on the UTAUT model. The sample consisted of 213 employees of companies of different sizes. We conclude that the use of IT contribute to more efficient use of resources, which would result in better organizational and perception of quality service by employees.
\end{abstract}

*Trabajo empírico-investigación. Investigación derivada de la Etapa 1 Proyecto Fondecyt $N^{\circ} 11100163$ período 2010-2012. Investigador responsable: Dra. Leslier Valenzuela Fernández. 
Keywords: Information Technology Adoption, Organizational Performance, Perception of Quality of Service.

\section{Resumen}

La presente investigación tiene como propósito (1) indagar en los factores que influirían en la adopción de TI, (2) estudiar las posibles influencias entre la adopción de TI, el desempeño organizacional y la percepción de calidad de servicio por parte de los empleados, (3) aportar evidencia empírica mediante la prueba de un modelo explicativo basado en el modelo UTAUT. La muestra consistió en 213 empleados de empresas de distintos tamaños. Se concluye que el uso de TI contribuiría a una mayor eficiencia en el uso de recursos, lo que se traduciría en mejores resultados organizacionales $y$ percepción de calidad de servicio por parte de los empleados.

Palabras clave: Adopción de tecnologías de información (TI), desempeño organizacional, percepción de calidad de servicio.

\section{Introducción}

Desde principios de los noventa los procesos de comunicación y análisis de información han tenido una evolución sin precedentes, principalmente gracias a la masificación del Internet (Calandra y Araya, 2009). Las herramientas tecnológicas se encuentran presentes en la mayor parte de las actividades cotidianas tanto de las personas como de las empresas, disminuyendo las barreras de la distancia y el tiempo y facilitando el desarrollo de un sinnúmero de tareas (Méndez y Monje, 2006).

En la actualidad, las tecnologías de la información (TI) son consideradas herramientas esenciales en la mejora de la economía de un país, derivado de los efectos significativos que tienen en la productividad de las empresas por la mejora de los procesos internos y del desempeño de los trabajadores (Brynjolfsson y Hitt, 1996; Lichtenberg, 1995; Shin, 2000). Ahora bien, la magnitud de estos 
efectos dependerá del nivel en que las TI se encuentren distribuidas y utilizadas (Oliveira y Martins, 2011). En este sentido, según Fraiha (2012), la adopción de la tecnología ocurre cuando una persona o grupo decide adquirir y comenzar a utilizar una tecnología en específico, ya sea para fines personales o de negocios.

Calandra y Araya (2009) mencionan que para que una TI sea considerada como tal debe cumplir 3 requisitos básicos: registrar información, comunicar y ser transportable. Ahora bien, como se aprecia en la definición anterior se consideran como TI las tecnologías que realizan distintas acciones de manera electrónica, no considerando otras TI como los libros y otras herramientas más tradicionales que cumplen con los tres requisitos mencionados con anterioridad. En esta investigación se tomó como unidad de estudio las TI que funcionan por medios electrónicos. Cabe destacar que a principios de este siglo el término TI derivó en N'TI, es decir, nuevas tecnologías de información (Calandra y Araya, 2009). Entre las TI destacan el teléfono, la radiofonía, la televisión, etcétera. Mientras que los celulares, el internet, el GPS y otras tecnologías corresponden a N'TI. Para efectos del presente estudio nos referiremos con el término TI tanto a las tecnologías pertenecientes a TI como a las N'TI, pues es ampliamente usado para referirse a ambas y es el término que más se utiliza de forma coloquial.

Diversos investigadores han intentado describir la adopción de TI, enfatizando en factores organizacionales (Azadegan y Teich, 2010) y cómo esta adopción influiría en la mejora del "desempeño organizacional" (Brynjolfsson y Hitt, 1996; Kohli y Devaraj, 2003; Mukhopadhyay et al., 1995; Curran, Matthew y Surprenant, 2003; Sethuraman y Parasuraman, 2005). Cabe destacar la discusión que se plantea en la literatura con relación a cómo medir "desempeño", tradicionalmente se han usado medidas como la rentabilidad, productividad y valor de mercado, pero en los últimos años se han agregado variables no financieras, porque permiten captar información del ambiente de negocios que las medidas financieras no pueden (Hoque, 2004). Las medidas no financieras también 
permiten analizar dimensiones de la posición competitiva, como la percepción que tienen los clientes o variaciones de la cuota de mercado (Kaplan y Norton, 1996; Vargas et al., 2003).

No obstante lo anterior, la adopción de las TI no está exenta de dificultades, ya que existe una serie de barreras que limitan la penetración, partiendo por el desconocimiento de ciertas tecnologías y/o de sus beneficios, creencia de costos crecientes y la falta de infraestructura y de formación (Campo et al., 2008).

Dado lo anterior, el propósito de la presente investigación es: (1) indagar en los factores que influyen en la adopción de TI por parte de las empresas, (2) estudiar las posibles influencias entre la adopción de TI, el desempeño organizacional y la percepción de calidad de servicio por parte de los empleados, (3) aportar evidencia empírica por la prueba de un modelo explicativo.

\section{Marco teórico}

La presente investigación tomó como referencia el modelo UTAUT de adopción de TI (Unified Theory of Acceptance and Use of Technology), desarrollado por Venkatesh, Morris y Davis en el 2003. La teoría basada en los recursos (TBR) para el desempeño organizacional y el modelo SERVQUAL de percepción de la calidad del servicio con el fin de desarrollar el modelo explicativo a probar.

\section{A. Adopción de las TI (Modelo UTAUT)}

El modelo UTAUT es una estructura integradora de distintos constructos existentes, basándose en diversas teorías y modelos pertenecientes a diversas áreas del conocimiento, entre los que destacan el Technology Acceptance Model (TAM) (Davis, 1989); la teoría de difusión de innovaciones (IDT) (Rogers, 1983); la teoría del comportamiento planeado-TPB (Ajzen, 1985); la teoría de la 
acción razonada (TRA) (Fishbein y Ajzen, 1975) y la teoría social cognitiva (SCT) (Bandura, 1986). El modelo UTAUT explica finalmente el impacto que tienen los constructos: "expectativas de desempeño, expectativas de esfuerzo y la influencia social" (Ajzen, 1985) sobre la intención de uso de tecnologías y el uso propiamente tal (Davis, 1989; Thompson et al., 1991; Moore y Benbasat, 1991). Según Venkatesh et al., 2003, las expectativas de desempeño se definen como "el grado en que un individuo cree que usar las TI le ayudará a aumentar el rendimiento en el trabajo"; las expectativas de esfuerzo corresponden al "nivel de percepción que tiene el individuo sobre la facilidad que poseen las TI para poder ser operadas de manera fácil" y la influencia social corresponde a la "influencia que ejercen otras personas sobre el individuo que debe tomar la decisión de utilizar una tecnología o no".

La intención de uso se define como una función ponderadora entre la actitud hacia el uso que refleja la positividad o negatividad de la aplicación de una tecnología y, la utilidad percibida que refleja la creencia de que utilizando la tecnología se mejorará el desempeño (Ajzen, 1985; Davis, 1989; Rogers, 1983).

Venkatesh y Morris (2003) y Moore y Benbasat (1991) han encontrado evidencia de que las expectativas de desempeño están relacionadas positivamente con la intención de uso. También ha sido probada por varios autores la relación positiva entre las expectativas de esfuerzo e intención de uso (Thompson et al., 1991; Ajzen, 1991; Davis, 1989; Fishbein y Azjen, 1975).

\section{B. Desempeño organizacional y teoría basada en los recursos (TBR)}

A partir de los años noventa, los investigadores han mostrado particular interés en intentar medir el impacto de la adopción de las TI en el desempeño organizacional, con el fin de justificar las 
inversiones en tecnología por parte de las empresas (Brynjolfsson y Hitt, 1993; Soh y Markus, 1995).

La evidencia existente hasta el momento no ha sido concluyente, algunos estudios han encontrado una relación positiva entre adopción de TI y desempeño (Brynjolfsson y Hitt, 1996; Kohli y Devaraj, 2003; Mukhopadhyay et al., 1995), versus otros que han encontrado evidencia de neutralidad o incluso una relación negativa o que la relación no es directa sino que por otras variables (Wade y Hulland, 2004). Entre las causas de estos resultados negativos o neutros se encuentran el retraso entre introducción de TI e impactos (Brynjolfsson y Hitt, 1993) y problemas de medición en las unidades de análisis de la empresa (Barua, 1995).

Ahora bien, la principal discusión en la literatura se refiere a cómo medir el constructo de "desempeño organizacional". Araya et al. (2007) distinguen 2 grandes grupos de mediciones: (1) mediciones financieras vs. mediciones no financieras y (2) mediciones objetivas vs. mediciones subjetivas. Por otra parte, Melville et al. (2004) proponen un modelo para analizar el impacto de las TI que comprende tres dominios. El primero es la empresa, en que confluyen tanto recursos de TI como recursos complementarios, para mejorar los procesos de negocio actuales o facilitar los procesos nuevos, los que posteriormente impactarán en el desempeño a nivel agregado de la empresa. El segundo dominio es el entorno competitivo. Y finalmente el entorno macroeconómico, y que comprende factores del país en que se desempeña la firma, como su infraestructura, sus políticas respecto de las TI, etcétera. Estos autores también distinguen los impactos a nivel interno, como reducción de costos, mayor rapidez en tareas versus los efectos organizacionales a nivel competitivo. Barua (1995), por su parte, desarrolla un modelo en que analiza los efectos en 2 niveles, efectos de "primer nivel" que corresponden a efectos operacionales y efectos de "alto nivel" que son aquellos que abarcan a la empresa como un todo. 
Debido a lo anterior, la presente investigación se enfocó en el impacto de las TI tanto a "nivel operacional" (procesos de negocio) como a "nivel organizacional" (Barua, 1991), considerando el desempeño en relación con los competidores y el dominio de la firma, es decir, la teoría basada en los recursos (Melville et al., 2004).

La TBR argumenta que las empresas poseen recursos que les permiten conseguir ventajas competitivas que incluso podrían ser de largo plazo (Barney, 1991; Grant, 1991; Penrose, 1959; Wernerfelt, 1984 en Wade y Hulland, 2004).

En este sentido, la TBR integra una perspectiva de administración con una perspectiva económica (Peteraf y Barney, 2003). Esta teoría se sustenta en 2 supuestos principales: (1) las firmas en una industria o grupo pueden ser heterogéneas respecto de los recursos estratégicos que controlan y (2) los recursos pueden no ser perfectamente móviles por medio de las firmas y la heterogeneidad puede ser de largo plazo (Barney, 1991; Peteraf, 1993). Estos supuestos son aplicables al uso de TI, pues a pesar de que las empresas pueden imitar recursos como software o hardware, el ajuste con la estrategia y el resto de los recursos complementarios marcará diferencias. La inmovilidad también puede ocurrir, pues a pesar de que una empresa puede eventualmente contratar a los trabajadores de otra, existen conocimientos no replicables, pues ni los mismos trabajadores entienden cómo se logran resultados superiores, al ser procesos complejos y que dependen de las circunstancias de la empresa, además para lograr ventajas como "habilidades de administración" se requiere tiempo (Barney, 1991; Mata et al., 1995).

Ahora bien, los recursos se clasifican en tres categorías: recursos de capital físico (la tecnología física, la planta y equipamiento, ubicación geográfica y acceso a materias primas); recursos de capital humano (el entrenamiento, la experiencia, el juicio, la inteligencia, las relaciones y la intuición de los administradores y trabajadores de la empresa) y recursos de capital 
organizacional (estructura formal de reporte, su planificación formal e informal, el control y la coordinación de sistemas, la relación informal entre grupos en una firma y de la firma con su ambiente) (Barney, 1991).

Aplicando lo anterior a las TI, este trabajo consideró dos grandes tipos de recursos TI (1) recursos TI y (2) recursos complementarios (Melville et al., 2004).

Los recursos TI incluyen tanto los recursos humanos TI como los recursos tecnológicos de TI. Dentro de los recursos humanos TI se distinguen las habilidades técnicas de TI, que corresponde a conocimientos de programación, mantención de equipos TI, trabajo con bases de datos, conocimiento de software específico, entre otros, y las habilidades directivas para administrar las TI, entre las que se encuentran la colaboración con unidades de negocio y dentro de la infraestructura se encuentran los elementos físicos TI, como hardware, software, redes, etcétera.

Respecto de los recursos complementarios TI, podemos decir que el hecho de que los recursos TI sean valiosos no es suficiente para que una ventaja competitiva sea sostenible, existiendo consenso en que su sola presencia no es suficiente para explicar las potencialidades de la empresa (Ventura, 1996), pues los competidores actuales o potenciales pueden adquirir las mismas herramientas, con lo que la posesión de estos recursos valiosos sería la norma en el mercado y no permitiría a una firma lograr un desempeño superior al de las demás empresas (Barney, 1991). Es por esto que se hace necesario considerar no solo los recursos TI, sino que también la interacción con otros recursos disponibles en la empresa, es decir, recursos complementarios (Bharadwaj, 2000; Araya et al., 2007); ejemplos de estos recursos son: las relaciones con los proveedores, el entrenamiento de TI, el diseño de los procesos de negocio, la orientación de equipo, el benchmarking, y la planificación de TI, la estructura organizacional, las políticas y reglas, las prácticas del lugar de trabajo, la cultura entre otros (Peteraf y Barney, 2003; Melville et al., 2004). 
Debido a lo anterior, las hipótesis de estudio que se desprenden son las siguientes:

H1: Los recursos TI influyen positivamente en el desempeño de los procesos de negocio.

H2: Los recursos complementarios influyen positivamente en el desempeño de los procesos de negocio.

H3: El desempeño de los procesos de negocio influye positivamente en la percepción de calidad de servicio por parte de los empleados.

H4: El desempeño organizacional influye positivamente en las expectativas de desempeño de nuevas tecnologías.

\section{Percepción de calidad de servicio (modelo SERVQUAL)}

El concepto de calidad de servicio ha sido objeto de análisis por muchos autores (Hunt, 1977; Oliver, 1980; Churchill y Suprenant, 1982; Giese y Cote, 2000) y se ha caracterizado por ser un concepto complejo. Fornell (1992) sostiene que la percepción de calidad es un fenómeno psicológico que está en función de la experiencia del consumidor con un producto o servicio. En términos generales podemos entender este concepto a base de tres aspectos concretos de una transacción: (1) la percepción de calidad es una respuesta emocional o cognitiva, (2) la respuesta pertenece a un foco particular (expectativa, producto, experiencia de consumo, etcétera) y (3) la respuesta ocurre en un tiempo específico, después del consumo basado en la experiencia acumulada, etcétera (Giese y Cote, 2000). 
Para las empresas que adoptan TI en sus operaciones, la calidad de servicio se comporta como un proxy para medir si efectivamente los consumidores perciben mejoras en la entrega del producto o servicio (Curran, Matthew y Surprenant, 2003). En este sentido, el objetivo debe estar orientado a que los clientes obtengan un resultado positivo de la diferencia entre la calidad percibida de la prestación y lo que realmente espera (Oliver, 1977).

En este contexto se utilizó el instrumento SERVQUAL de medición de calidad de servicio (Parasuraman et al., 1988), el que está compuesto por las dimensiones: confianza, seguridad, responsabilidad, tangibilidad y empatía (Fitzsimmons y Fitzsimmons, 2000) (ver tabla 1). Ahora bien, cabe destacar que este constructo se midió mediante las "percepciones de calidad de servicio de los trabajadores de la empresa".

Lo anterior nos lleva a probar las siguientes hipótesis:

H5: La intención de uso influye positivamente en la percepción de calidad de servicio por parte de los empleados.

H6: La percepción de calidad de servicio por parte de los empleados influye positivamente en el desempeño organizacional.

A partir de lo anterior, a continuación se propone el modelo explicativo a probar (ver figura 1). 
Tabla 1

Definiciones y dimensiones de la calidad de servicio

\begin{tabular}{|c|c|}
\hline DIMENSIÓN & DEFINICIÓN \\
\hline Confianza & $\begin{array}{l}\text { Consiste en prestar el servicio en forma fiable y cuidadosa. } \\
\text { El desempeño de un servicio confiable forma parte de las } \\
\text { expectativas del cliente y significa que el servicio se } \\
\text { prestará en el plazo establecido, sin cambios ni errores. }\end{array}$ \\
\hline Seguridad & $\begin{array}{l}\text { Se refiere a los conocimientos, atención y habilidades } \\
\text { mostradas por los empleados para inspirar credibilidad y } \\
\text { confianza. Competencia para brindar el servicio, cortesía y } \\
\text { respeto por el cliente, comunicación efectiva con el cliente } \\
\text { y mostrar que el empleado está interesado en lo mejor para } \\
\text { el cliente. }\end{array}$ \\
\hline Responsabilidad & $\begin{array}{l}\text { Disposición de los empleados para ayudar a los usuarios a } \\
\text { proveerlos de un servicio rápido. Al surgir fallas, la } \\
\text { capacidad de resolverlas rápidamente y con profesionalismo } \\
\text { puede tener un impacto positivo de calidad. }\end{array}$ \\
\hline Tangibilidad & $\begin{array}{l}\text { Apariencia de las instalaciones físicas, equipo, personal y } \\
\text { materiales de comunicación. La condición del ambiente es } \\
\text { una evidencia tangible del cuidado y la atención a los } \\
\text { detalles exhibidos por el proveedor del servicio. También } \\
\text { puede extenderse a la conducta de otros clientes que estén } \\
\text { utilizando el servicio. }\end{array}$ \\
\hline Empatía & $\begin{array}{l}\text { Se refiere al esfuerzo por entender la perspectiva del } \\
\text { usuario mediante la atención individualizada, se refiere a la } \\
\text { accesibilidad, sensibilidad y esfuerzo para entender las } \\
\text { necesidades del cliente. }\end{array}$ \\
\hline
\end{tabular}

FUENTE: Fitzsimmons y Fitzsimmons, 2000. 


\section{Figura 1}

Modelo explicativo

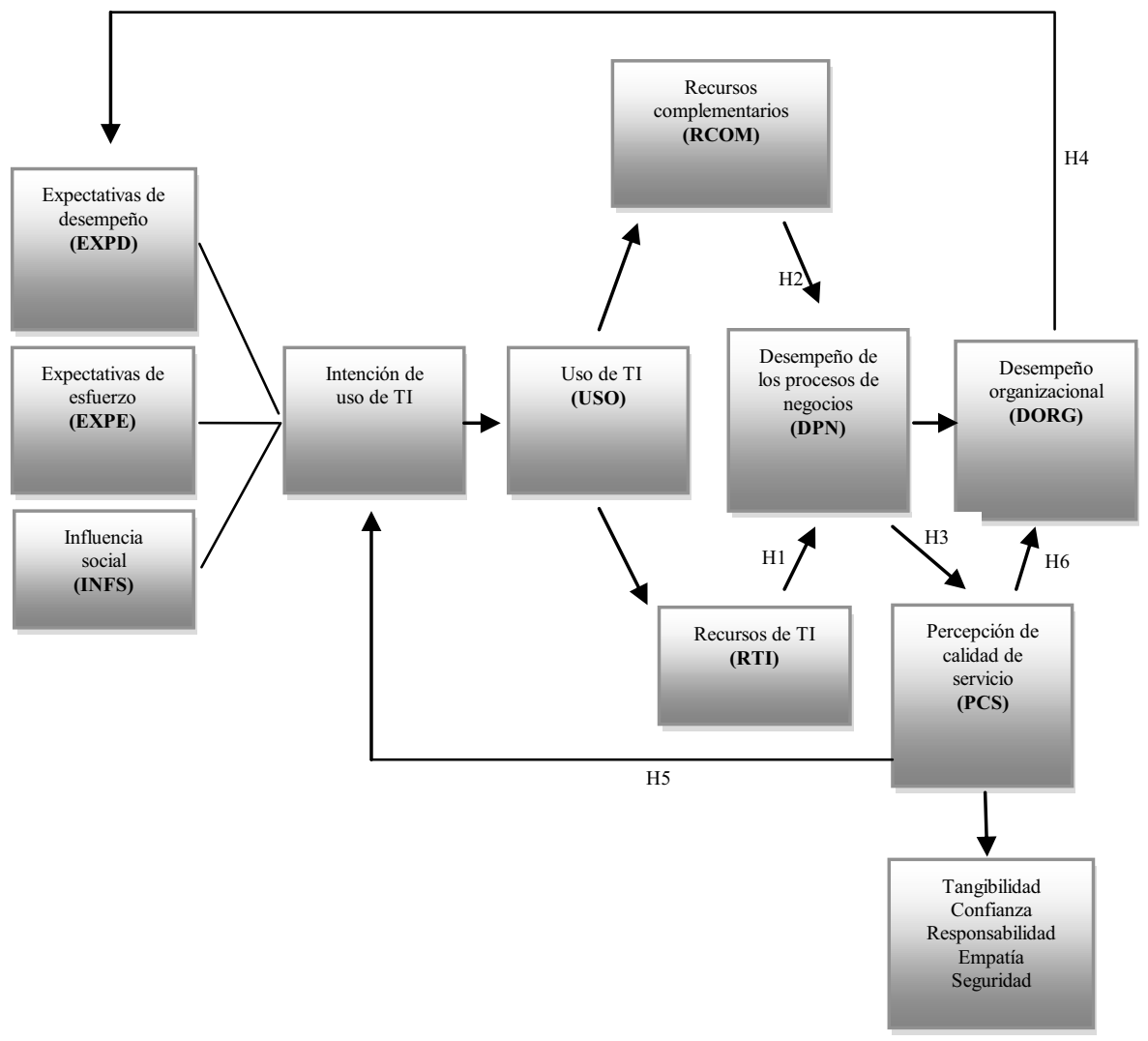




\section{Metodología}

Se realizó un análisis cuantitativo transversal simple con una estrategia de obtención de datos vía presencial. El instrumento aplicado fue un cuestionario autoadministrado con 43 indicadores, los que se midieron por medio de una escala Likert de 7 puntos, donde 1 = "totalmente en desacuerdo" y $7=$ "totalmente de acuerdo"; compuesto de los constructos: intención de uso de TI, uso de TI, percepción de calidad de servicio por parte de los empleados, recursos TI, recursos complementarios, desempeño de los procesos de negocio, desempeño organizacional. Previo a la realización de las encuestas se realizó un pretest del instrumento con el fin de depurarlo. Los criterios utilizados para examinar la unidimensionalidad, validez convergente y fiabilidad de los constructos fueron: Kayser-Meyer-Olkin mayor que 0,6, Eigenvalue mayor que 1, el test de esfericidad de Bartlett menor que 0,5, cargas factoriales mayores a 0,7 , y alpha de Cronbach mayor que 0,7.

La muestra fue del tipo no probabilístico por conveniencia, la que consistió en 213 personas. El universo objetivo correspondió a empleados y mandos medios de empresas de distintos tamaños en Chile (pequeña, mediana y gran empresa).

\section{Resultados}

A. Caracterización de la muestra

La muestra consistió en 213 encuestados, conformado por un número equitativo de hombres y mujeres, representados por $49,3 \% \mathrm{y}$ $50,7 \%$, respectivamente. En su mayoría personas menores de 34 años $(66,7 \%)$, con un nivel dominante de estudios universitarios completos $(33,3 \%)$ e incompletos $(23,9 \%)$ y que trabajan de forma dependiente $(68,6 \%)$. 
La caracterización de las empresas en donde se desempeñan los encuestados fue de $58,30 \%$ en microempresa, pequeña empresa y mediana empresa y $41,7 \%$ en grandes empresas.

\section{B. Análisis de fiabilidad y análisis factorial conjunto}

El análisis de fiabilidad conjunto entregó un alfa de Cronbach para todo el modelo de 0,956 , mostrando consistencia interna. Además, mostró un nivel de adecuación muestral KMO de 0,906 y el test de esfericidad de Bartlett rechazó la hipótesis nula de diagonalidad de las variables.

El análisis factorial exploratorio arrojó nueve factores que explicaron más del $70 \%$ de la varianza. Al realizar un análisis individual por constructo se observó que todos superaron el nivel mínimo de alpha de Cronbach exigido y se agruparon en un solo factor (unidimensionalidad).

\section{Comprobación de hipótesis}

Se analizaron las correlaciones con el fin de observar la posible influencia o relación (positiva o negativa) entre los diferentes constructos (ver tabla 2).

El constructo de intención de uso de TI se compone de las expectativas de desempeño, influencia social y expectativas de esfuerzo. Las correlaciones calculadas están por sobre los 0,3 $(0,499,0,369$ y 0,364 , respectivamente), es decir, existiría una relación positiva entre las variables. Por tanto, la intención de uso de las TI podría explicar el uso propiamente tal de estas, lo que coincide con la evidencia previa. 


\section{Tabla 2}

Matriz de correlaciones

\begin{tabular}{|c|c|c|c|c|c|c|c|c|c|}
\hline & EXPD & INFS & EXPE & USO & PCS & DPN & RCOM & RTIC & DORG \\
\hline EXPD & 1,000 & 0,362 & 0,476 & 0,499 & 0,603 & 0,646 & 0,373 & 0,333 & 0,360 \\
\hline INFS & 0,362 & 1,000 & 0,324 & 0,369 & 0,550 & & 0,510 & 0,509 & 0,488 \\
\hline EXPE & 0,476 & 0,324 & 1,000 & 0,364 & 0,478 & 0,502 & 0,436 & 0,374 & 0,315 \\
\hline USO & 0,499 & 0,369 & 0,364 & 1,000 & 0,444 & 0,539 & 0,546 & 0,519 & 0,530 \\
\hline PCS & 0,603 & 0,550 & 0,478 & 0,444 & 1,000 & 0,675 & 0,493 & 0,441 & 0,477 \\
\hline DPN & 0,646 & 0,475 & 0,502 & 0,539 & 0,675 & 1,000 & 0,635 & 0,617 & 0,699 \\
\hline RCOM & 0,373 & 0,510 & 0,436 & 0,546 & 0,493 & 0,635 & 1,000 & 0,755 & 0,702 \\
\hline RTIC & 0,333 & 0,509 & 0,374 & 0,519 & 0,441 & 0,617 & 0,755 & 1,000 & 0,708 \\
\hline DORG & 0,360 & 0,488 & 0,315 & 0,530 & 0,477 & 0,699 & 0,702 & 0,708 & 1,000 \\
\hline
\end{tabular}

Todos con $\mathrm{p}<0,0$.

El nivel de correlación entre los recursos TI de las empresas y el desempeño de los procesos de negocio es relativamente alta $(0,617)$. Por tanto, la gestión de la calidad y el buen uso de los recursos tecnológicos en las empresas tendrían un efecto positivo en la eficiencia de sus procesos internos.

La relación entre los recursos complementarios y el desempeño de los procesos de negocio alcanza 0,635. Esto implicaría que al gestionar eficientemente los recursos complementarios con los que cuentan las empresas se podrían lograr sinergias que impacten en la eficiencia del desempeño de los procesos de negocio.

Respecto de la posible relación entre los constructos desempeño de los procesos de negocio y desempeño organizacional resultó ser positiva $(0,699)$, es decir, la eficiencia en el desempeño de los procesos de negocio internos podría impactar de forma positiva al desempeño organizacional.

La correlación existente entre el desempeño de los procesos de negocio y la percepción de calidad de servicio por parte de los empleados correspondió a 0,672 , esto podría implicar que posibles 
aumentos en la eficiencia en los procesos de negocio permitiría que los empleados entreguen productos y servicios a sus clientes con mayor rapidez y mejorar la relación entre las partes.

El desempeño organizacional correlaciona positivamente con las expectativas de desempeño de otras tecnologías en una cuantía de 0,360. Esto podría implicar que mientras más alto sea el desempeño alcanzado por las empresas al incorporar una nueva tecnología, dicha empresa se encontrará en un escenario favorable y en mejores condiciones para adaptarse a "otras" nuevas tecnologías que contribuyan en el desempeño.

La percepción de calidad de servicio por parte de los empleados afectaría positivamente a las expectativas de desempeño, influencia social y expectativas de esfuerzo. Estas relaciones determinan el nivel de intención de uso $(0,603,0,550$ y 0,478), respectivamente. Esto implica que mayores niveles de calidad de servicio entregada por los empleados podrían influir en la adopción y uso de tecnologías y afectaría positivamente en las expectativas de esfuerzo, ya que sería más fácil cumplir con las exigencias de los clientes y por tanto disminuir el esfuerzo para cumplir este objetivo.

La percepción de calidad de servicio por parte de los empleados ejercería una influencia positiva sobre el desempeño organizacional $(0,477)$. Esto debido a que componentes de la calidad de servicio como tangibilidad, seguridad y confianza son factores que los empleados y mandos medios encuestados estiman tienen una alta importancia para los consumidores, lo que aumentaría la probabilidad de una repetición de compra y de una relación más a largo plazo, lo que afectaría positivamente el desempeño de la empresa. 


\section{Conclusiones}

El propósito perseguido por el presente estudio fue: (1) indagar en los factores que influyen en la adopción de TI por parte de las empresas, (2) estudiar las posibles influencias entre la adopción de TI con el desempeño organizacional y la percepción de calidad de servicio por parte de los empleados, (3) aportar evidencia empírica mediante la comprobación de un modelo explicativo.

Los resultados del estudio empírico indicaron que las variables del modelo propuesto podrían contribuir a explicar la influencia positiva de las TI en el desempeño y la percepción de calidad de servicio por parte de los empleados.

Respecto de la influencia de la percepción de calidad de servicio por parte de los empleados en la intención de uso de TI se encontraron correlaciones positivas con los subconstructos que componen la intención de uso.

En el escenario actual en que se desenvuelven las empresas, el que se ha tornado cada vez más competitivo producto de la mayor integración económica y la progresiva disminución de las barreras al comercio entre países, acompañado de que los clientes son cada vez más informados, exigentes y menos leales a las empresas, la lealtad del consumidor y la tasa de recompra, determinarían la rentabilidad y crecimiento del negocio (Palmatier et al., 2006). Según Valenzuela (2010), las intenciones de compra del cliente y compras reales dependerán tanto de la calidad del servicio, la satisfacción y la confianza que se genere con el proveedor, debido principalmente a que los clientes formarían sus opiniones y juicios de la empresa basados en factores económicos y no económicos, asociados con el producto o servicio (Zeithaml et al., 1993). En este contexto, el uso de TI se presenta como una interesante alternativa que permitiría hacer frente a estos desafíos, ya que por un lado contribuirían a una mayor eficiencia en el uso de los recursos, lo que se traduciría en mejores resultados organizacionales y además impactaría positivamente en la percepción de calidad de servicio por parte de 
los empleados, al facilitar el intercambio cliente-empresa como por ejemplo: la posibilidad de realizar compras sin moverse de su hogar, conocer el estado del envío de productos, reducir los tiempos de despacho, etcétera, lo que podría ayudar a tener clientes más leales y a la vez disminuir los costos de fidelización, retención y captación de clientes.

Chile se encuentra bien posicionado respecto de la adopción de TI, pero al compararse con países desarrollados se observan brechas en varios aspectos (conectividad e infraestructura en tecnologías, aspectos legales, sociales, culturales, adopción de TI por parte de consumidores y empresas). Por ejemplo el Digital Economy Ranking ${ }^{1}$ ubicó a Chile en el puesto número 30 en el 2010, con una puntuación de 6,39 (de un máximo de 10), cayendo 0,10 puntos respecto del año anterior.

De los resultados de esta investigación se desprende que los recursos complementarios serían relevantes para lograr buenos resultados al adoptar las TI, por lo que las empresas deberían poner atención a este punto y adaptar su cultura, estructura, prácticas, etcétera, para apoyar la implementación, pues si no se tiene cuidado con esto se reduciría la probabilidad de tener resultados positivos, lo que aumentaría los costos para las empresas.

Las limitaciones del presente estudio se relacionan con la falta de acceso a la información de las empresas de manera directa, debido a que las encuestas fueron aplicadas a empleados y mandos medios que en muchos casos tienen información incompleta, ya que su conocimiento está limitado principalmente al área o subsector en que se desempeñan y para responder las preguntas deben recurrir a sus percepciones, lo que constituye información subjetiva. Otra limitación del estudio es la no inclusión de variables externas al

${ }^{1}$ Índice de conectividad e infraestructura en tecnologías, ambiente de negocios, entorno social y cultural, entorno legal, visión y políticas públicas y adopción de TI por parte de los consumidores y empresas. Desarrollado por Economist Intelligence Unit y el Institute for Business Value. 
dominio de la empresa (Melville et al., 2004), como variables específicas de cada país, como aspectos culturales, infraestructura, niveles educacionales, etcétera, y características propias del mercado como la regulación, los cambios tecnológicos y dominio del entorno competitivo.

La utilización de una escala genérica como la SERVQUAL produce sesgos al someterla a un estudio general, cada sector de la economía posee distintas características y se comportan de manera diferente teniendo muchas veces otras dimensiones, además la satisfacción del cliente fue medida por medio de las percepciones de los trabajadores (oferta), principalmente por un asunto de factibilidad.

En futuros estudios se recomienda la realización de un seguimiento longitudinal del proceso de implementación de las TI con el fin de observar la interacción que se produce entre los recursos TI y los recursos complementarios, analizando las barreras y los factores catalizadores para lograr una buena implementación. Además sería recomendable medir la satisfacción del cliente (demanda) y así tener una real impresión de las posibles divergencias que existirían con la percepción de calidad de servicio por parte de la oferta. También sería interesante analizar las diferencias de implementación de tecnologías según tipo de empresa dependiendo de su estrategia de negocios específica. 


\section{Referencias}

AJZEN, I. (1985), "From Intentions to Behavior: A Theory of Planned Behavior", in I. J. K. J. B. (eds.), Action-Control: From Cognition to Behavior (Spinger, Heidelberg), pp. 11-39.

AJZEN, I. (1991), "The theory of planned behavior", Organizational Behavior and Human Decision Processes, Vol. 50, pp. 179-211.

Araya, S., J. Chaparro, A. Orero y H. Joglar (2007), "Sistemas y Tecnologías de Información y su relación con el Desempeño de Instituciones Universitarias en el ámbito de Gestión Institucional: un estudio desde la Teoría de Recursos y Capacidades", International Conference on Industrial Engineering \& Industrial Management - CIO 2007.

AZAdEGAN, A. y J. TEICH (2010), "Effective benchmarking of innovation adoptions: A theoretical framework for e-procurement technologies", Benchmarking: An International Journal, Vol. 17, № 4, pp. 472-490.

BANDURA, A. (1986), "Social foundations of thought and action: A social cognitive theory", Englewood Cliffs, NJ: Prentice-Hall.

BARNEY, J. (1991), "Firm resources and sustained competitive advantage", Journal of Management, Vol. 17, pp. 99-120.

Barua, A., H.C. Kriebel y T. Mukhopadhyay (1991), “An economic analysis of strategic information technology investment", MIS Quaterly.

BARUA, A., H.C. Kriebel y T. MuKhopadhyAY (1995), "Information technologies and business value: an analytic and empirical investigation", Information Systems Research, Vol. 6, № 1, pp. 3-23.

BHARADWAJ, A. (2000), "A resource-based perspective on information technology capability and firm performance: an empirical investigation”, MIS Quaterly, Vol. 24, No 1, pp. 169-196.

BRYNJOLFSSON, E. y L. HITT (1993), "Is Information Systems Spending Productive? New Evidence and New Results", in Proceedings of the Fourteenth International Conference on Information Systems, J.I. DeGross, R.P. Bostrom, and D. Robey (eds.), Orlando, Florida. 
BRYNJOLFSSON, E. y L. HitT (1996), "Paradox Lost? Firm-Level Evidence on the Returns to Information Systems Spending”, Management Science, Vol. 42, $\mathrm{N}^{\mathrm{o}} 4$, pp. 541-558.

Calandra, P. y M. Araya (2009), "Conociendo las TIC", Santiago, Libro lanzado por la Universidad de Chile en cooperación con INOVA Chile y Corfo, Facultad de Ciencias Agronómicas.

CAMPo, S., N. Rubio y M. YagüE (2008), "Beneficios y costes del uso de las TIC para las empresas minoristas de distribución de electrodomésticos", Distribución y Consumo, Vol. 102, pp. 94-101.

Churchill A. JR. and C. Surprenant (1982), "An Investigation into the Determinants of Customer Satisfaction", Journal of Marketing Research, Vol. 19, pp. $491-504$

Curran, J., M. Matthew y C. Surprenant (2003), "Intentions to Use SelfService Technologies: A Confluence of Multiple Attitudes", Journal of Service Research, Vol. 5, No 3, pp. 209-224.

DAVIS, F. (1989), "Perceived Usefulness, Perceived Ease of Use, and User Acceptance of Information Technology", MIS Quarterly, Vol. $13 \mathrm{~N}^{\mathrm{o}} 3$, pp. 319-339.

FISHBEIN, M. y I. AJZEN, (1975), "Belief, attitude, intention and behavior", Reading, M.A.: Addison-Wesley.

Fitzsimmons, J. y M. Fitzsimmons (2000), "New Service Development Creating Memorable Experiences", Sage Publications, Thousand Oaks, CA.

ForNELL, C. y Y. Yi (1992), “Assumptions of the Two-Step Approach to Latent Variable Modeling”, Sociological Methods \& Research, Vol. 20, No 3, pp. 291-320.

FRAIHA, S. (2012), "The effects of reference disciplines on technology adoption research", International Journal of Business Research, Vol. 12, $\mathrm{N}^{\mathrm{o}} 2$, pp. 145-154.

GIESE, J. y J. CotE (2000), "Defining consumer satisfaction", Journal of the Academy of Marketing Science. 
GRANT, R. M. (1991), “The Resource-Based Theory of Competitive Advantage: Implications for Strategy Formulation", California Management Review, Vol. 33, $\mathrm{N}^{\mathrm{o}} 1,114-135$.

HoQue, Z. (2004), “A contingency model of the association between strategy, environmental uncertainty and performance measurement: Impact on organizational performance", International Business Review, Vol. 13, pp. $485-502$.

HunT, H. (1977), “Conceptualization and Measurement of Customer Satisfaction and Dissatisfaction”, Cambridge: Marketing Science Institute.

KAPLAN, R. y D. NORTON (1996), "Using the balanced scorecard as a strategic management system", Harvard Business Review, Vol. 74, No 1, pp. 75-85,

KoHli, R. y S. DevaraJ (2003), "Measuring information technology payoff: A meta-analysis of structural variables in firm level empirical research", Information Systems Research, Vol. 14, No 2, pp. 127-145.

LiChtenberG, F. (1995), “The Output Contributions of Computer Equipment and Personnel: A Firm- Level Analysis", Economics of Innovation and New Technology, Vol.3, pp. 201-217.

MATA, F., W. FUERST y J. BARNEY (1995), "Information Technology and sustained competitive advantage: a resource-based analysis", MIS Quarterly, Vol. 19, No 4, pp. 487-505.

Melville, N., K. Kraemer y V. Gurbaxani (2004), "Review: information technology and organizational performance: an integrate model of IT business value", MIS Quarterly, Vol. 28, № 2, pp. 283-322.

MÉnDEZ, V. y J. Monge (2006), "Las TIC en un entorno latinoamericano de educación a distancia: la experiencia de la UNED de Costa Rica", RED. Revista de Educación a Distancia, Vol. 15. En línea en: http://www.um.es/ead/red/15/.

Moore, G. y I. BenbasAt (1991), "Development of an Instrument to Measure the Perceptions of Adopting an Information Technology Innovation", Information Systems Research, Vol. 2(3), pp. 192-222. 
Mukhopadhyay, T., S. KeKre y S. Kalathur (1995), "Business value of information technology: A study of electronic data interchange", MIS Quart, Vol.19, No 2, pp. 137-156.

Oliveira, T. y M. MARTINS (2011), "Literature Review of Information Technology Adoption Models at Firm Level", Electronic Journal of Information Systems Evaluation, Vol. 14, No 1, pp. 110-121.

Oliver, R. (1977), "Effect of Expectation and Disconfirmation on Postexposure Product Evaluations: An Alternative Interpretation", Journal of Applied Psychology, Vol. 62, pp. 480-486.

Oliver, R. L. (1980), “A Cognitive Model of the Antecedents and Consequences of Satisfaction Decisions", Journal of Marketin g Research, Vol. 18, pp. $460-469$.

Palmatier, R., R. DANT, D. Grewal y K. Evans (2006), "Factors Influencing the Effectiveness of Relationship Marketing: A Meta-Analysis", Journal of Marketing, Vol. 70, No 10, pp. 136-53.

PARASURAMAN, A., V. ZeITHAML y L. BERRY (1988), "SERVQUAL: A multiple item-scale for measuring consumer perceptions of service quality", Journal of Retailing, Vol. 64, $\mathrm{N}^{\mathrm{o}}$ 1, pp. 12-37.

PETERAF, M. y J. BARNEY (2003), "Unraveling the resource-based tangle" Managerial and Decision Economics, Vol. 24, No 4, pp. 309-320.

Peteraf, M. (1993), “The Cornerstones of Competitive Advantage: A ResourceBased View", Strategic Management Journal, Vol. 14, No 3, pp. 179-91.

Rogers, V. (1983), "Diffusion of Innovations", Third Ed., The Free Press, New York.

Sethuraman, R. y A. Parasuraman (2005), "Succeeding in the Big Middle through technology", Journal of Retailing, Vol. 81, No 2, pp. 107-111.

SHIN, I. (2000), "Use of information networks and organizational productivity: Firm-level evidence in Korea", Economics of Innovation and New Technology, Vol. 9, № 5, pp. 447-463. 
SoH, C. y M. MARKus (1995), "How IT Creates Business Value: A Process Theory Synthesis", in Proceedings of the Sixteenth International Conference on Information Systems, J. I. DeGross, G. Ariav, C. Beath, R. Hoyer, and C. Kemerer (eds.), Amsterdam, 1995, pp. 29-41.

Thompson, R., C. Higgins y J. Howell (1991), "Personal Computing: Toward a Conceptual Model of Utilization", MIS Quarterly, Vol. 15, N ${ }^{\circ}$, pp. 124-143.

VALENZUELA, L. (2010), "Marketing orientado al cliente y ética empresarial: efectos sobre el valor de la cartera", Estudios de Administración, Vol. 17. $\mathrm{N}^{\mathrm{o}} 2$, pp. 1-24.

VARGAS, A., M. HeRnÁNDEZ y S. BRUQUe (2003), "Determinants of information technology competitive value, Evidence from a western European industry", Journal of High Technology Management Research, Vol. 14, pp. 245-268.

Venkatesh, V., M.G. Morris, G.B.Davis y F.D. Davis (2003), "User Acceptance of Information Technology: Toward a Unified View”, MIS Quarterly, Vol. 27, No 3, 425-478.

Venkatesh, V. y M.G. Morris (2000), “Why Don’t Men Ever Stop to Ask for Directions? Gender, Social Influence, and Their Role in Technology Acceptance and Usage Behavior", MIS Quarterly, Vol. 24, N ${ }^{\circ}$, pp. 115-139.

VENTURA, J. (1996), “Análisis dinámico de la estrategia empresarial: Un ensayo interdisciplinar", Oviedo: Universidad de Oviedo.

WADE, M. y J. Hulland (2004), "The resource-based view and information systems research: review, extension, and suggestions for future research", MIS Quarterly, Vol. 28, № 1, pp. 107-142.

Zeithaml, V.A., L. Berry y A. PARASURAmAn (1993), "The Nature and Determinants of Customer Expectations of Service", Journal of the Academy of Marketing Science, Vol. 21, No 1, pp. 1-12. 